\title{
Developments of a new data acquisition system at ANNRI
}

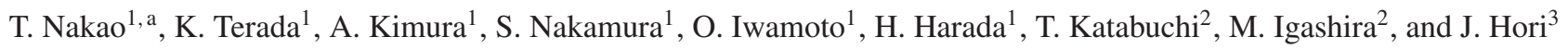 \\ 1 Nuclear Science and Engineering Center (NSEC), Japan Atomic Energy Agency (JAEA), Tokai-mura Naka-gun, \\ Ibaraki 319-1195, Japan \\ 2 Laboratory for Advanced Nuclear Energy, Tokyo Institute of Technology, 2-12-1 Ookayama, Meguro-ku, Tokyo 152-8550, Japan \\ 3 Kyoto University Research Reactor Institute, Kumatori-cho, Sennan-gun, Osaka 590-0494, Japan
}

\begin{abstract}
A new data acquisition system (DAQ system) has been developed at the Accurate Neutron-Nucleus Reaction Measurement Instrument (ANNRI) facility in the Japan Proton Accelerator Research Complex, Materials and Life Science Experimental Facility (J-PARC/MLF). DAQ systems for both the Ge detector system and the Li-glass detector system were tested by using a gold sample. The applicability of the time-offlight method was checked. System performance was evaluated on the basis of digital conversion nonlinearity, energy resolution, multi-channel coincidence and dead time.
\end{abstract}

\section{Introduction}

In J-PARC [1], an increase of the beam power by more than a factor of 20 was achieved in 2015 , to approximately $500 \mathrm{~kW}$, up from the first beam shot in 2009 of $20 \mathrm{~kW}$ [2]. This increase in beam power increases the rate at which users obtain experimental data.

In the ANNRI facility [3], two different types of $\gamma$-ray detector systems are operated for neutron capture crosssection measurements. One is a high-purity germanium detector system (Ge system), which has very fine $\gamma$-ray energy resolution; the other is a NaI detector system (NaI system) with the capability of measurement in a wide neutron-energy range. As the current DAQ system [4] for the Ge system was designed in 2009 for the lower beam power, the counting rate performance is almost saturated for the current beam power. In order to adapt to the current beam power, a new DAQ system has been developed.

In addition, a new Li-glass neutron detector system (Li-glass system) for total cross-section measurements was recently installed. A new DAQ system for the Li-glass detector must be developed, because the current DAQ system cannot be applied for the Li-glass detector signals as is.

\section{DAQ system specifications}

In order to measure total cross-sections, the beam must be well collimated on the upstream and downstream sides of the measuring samples to reduce small angle scattering effects. In ANNRI, two collimator systems were installed. One is a rotary collimator on the upstream side of the Ge system. The other is an intermediate collimator between the Ge and NaI systems. As there is no remaining space to set an additional collimator system on the downstream side of the NaI system, the measured sample must be located between the rotary collimator and the intermediate collimator in the case of total cross-

a e-mail: nakao.taro@jaea.go.jp section measurements. As the geometrical requirement for total cross-section measurements is in good agreement with that for capture cross-section measurements using the Ge system, a simultaneous measurement of the total and capture cross-sections can be performed if the DAQ system allows data to be simultaneously taken from different locations.

For the purpose of flexible simultaneous measurements, the DAQ system is designed using the TCP/IP protocol based client-and-server model. The role of the client-side application is to gather data from each ADC and send it to the server-side application. The serverside receives data from each client, builds one event data block, and saves it to disk. Each client is controlled by a common signal issued from the server-side application. As a consequence of the client-and-server model design, the DAQ system can accept additional ADC systems flexibly, become a client, and connect to the server-side.

Inside the client-side application, a core module is written in the $\mathrm{C}++$ programming language which is based on an object-oriented model. In basic usage, one object corresponds to one ADC board. As the client-side application back end makes a parallel routine for each object automatically, this DAQ system runs with linear scalability to the number of ADC boards up to CPU core number minus one (one core is used by DAQ system) without any modification of the user-side code.

To digitize the detector signals, two different types of ADCs are used. CAEN v1724 [5] 14-bit 100-MHz peakhold ADC boards are characterized by fine pulse height digitizing resolution, which is suitable for Ge systems. For the Li-glass system, which has a fast response signal output, CAEN v1720 12-bit $250-\mathrm{MHz}$ charge-sensitive ADCs are prepared. Optical link connections are used for data transfer from the ADC boards to the PC using a CAEN A3818 PCI express board. The maximum data transfer rate is about $80 \mathrm{MB} / \mathrm{s} /$ fibre.

Signals from the J-PARC beam monitor were used in order to measure time-of-flight events. Trigger signals

(C) The Authors, published by EDP Sciences. This is an Open Access article distributed under the terms of the Creative Commons Attribution License 4.0 (http://creativecommons.org/licenses/by/4.0/). 


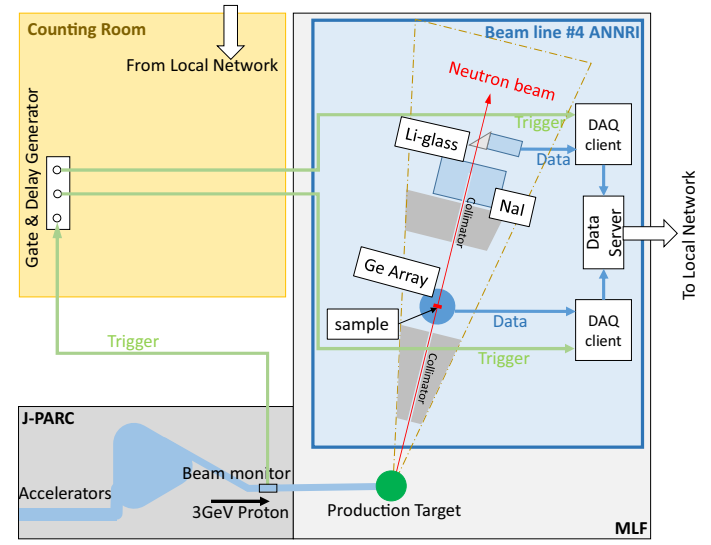

Figure 1. Schematic view of the J-PARC/MLF/ANNRI facility and the data flow of the DAQ system.

issued from J-PARC were distributed to the ANNRI counting room and distributed to an appropriate number of signals. As the trigger signal reaches the facility several microseconds after the fastest proton events (so called "gamma-flash"), each trigger is delayed one cycle length minus several microseconds, so that gamma-flash events arrive immediately after the trigger signal reset is received, shifted by one cycle.

Figure 1 shows the schematic diagram of the JPARC/MLF/ANNRI facility and the data flow of the DAQ system.

\section{DAQ system performance}

A test of the new DAQ system was performed using standard $\gamma$-ray sources and the ANNRI beam line. Applicability of the time-of-flight method was checked by using a ${ }^{197} \mathrm{Au}$ sample of thickness $100 \mu \mathrm{m} .{ }^{27} \mathrm{Al}(\mathrm{n}, \gamma){ }^{28} \mathrm{Al}$ neutron capture events were used for estimating the ADC non-linearity and energy resolution performance.

\subsection{Ge detector system}

In the ANNRI facility, the array of Ge detectors includes two clusters of Ge-detectors and a total of 20 BGO crystals. Each cluster of Ge-detectors consists of $7 \mathrm{Ge}$ crystals, for a total of 14 crystals. Signals from the BGO detectors were summed together into 4 channels, before being input into Ortec 474 timing filter amplifiers. Additionally, 8 coaxial type Ge detectors are located around the sample position. The DAQ system for the Ge detector system is required to obtain 26 signals in total.

The time-of-flight method applicability was checked by using a ${ }^{197} \mathrm{Au}$ sample of thickness $100 \mu \mathrm{m}$. Figure 2 shows the time-of-flight spectrum and the $\gamma$-ray spectrum taken by v1724 ADCs. The first resonance of $\mathrm{Au}$ at $4.89 \mathrm{eV}$ [6] and other resonance structures followed by the neutron capture events were clearly observed.

The ADC non-linearity and photo-peak energy resolution were checked in the $100 \mathrm{keV}$ to $8 \mathrm{MeV}$ range using a ${ }^{152} \mathrm{Eu}$ standard $\gamma$-ray source and ${ }^{27} \mathrm{Al}(\mathrm{n}, \gamma) \gamma$-rays. Typically $\pm 0.5 \mathrm{keV}$ linearity and $3 \sim 8 \mathrm{keV} @ 1000 \mathrm{keV}$ energy resolution were obtained.

The coincidence information is required for some analysis, for example $\gamma-\gamma$ analysis. In order to perform coincidence analysis, time-stamp information and the
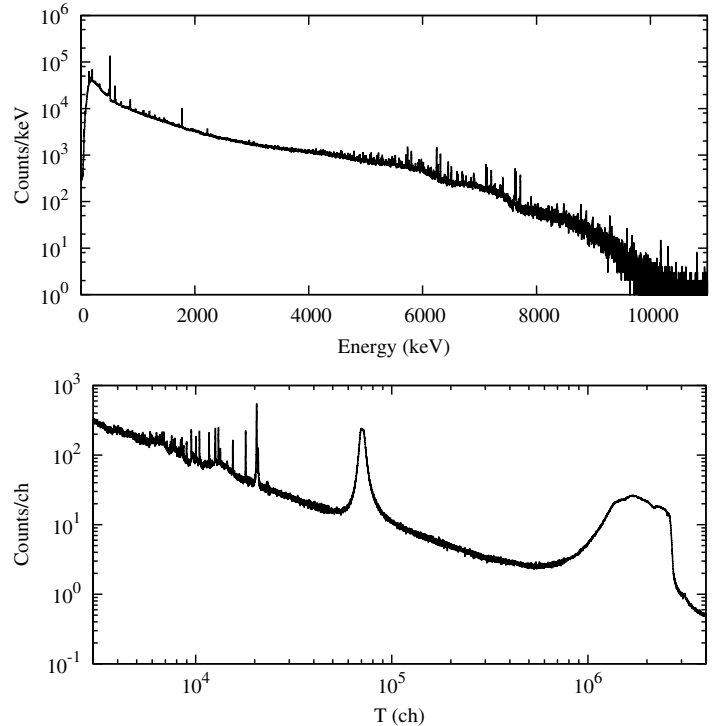

Figure 2. The $\gamma$-ray spectrum (upper figure) and the time-offlight spectrum (lower figure) of a Ge detector using a $100-\mu \mathrm{m}$ thick ${ }^{197} \mathrm{Au}$ sample.

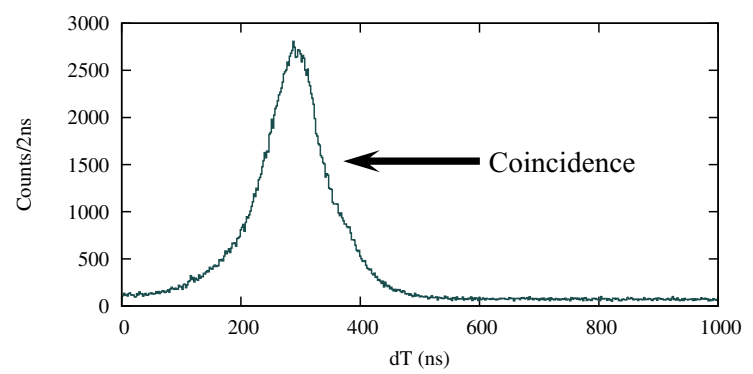

Figure 3. The time difference between a Ge event and a BGO event. The peak in the spectrum indicates coincidental events in which a single reaction results in multiple events in both $\mathrm{Ge}$ and BGO crystals.

trigger numbering method can be used. Using this information, events in coincidence timing can be distinguished in offline software analysis. Figure 3 shows an example of offline analysis of coincidence between the $\mathrm{Ge}$ and BGO signals. The time difference between one channel of a Ge event and the paired BGO event is shown. A clear peak structure caused by coincidental events was observed.

\subsection{Li-glass detector system}

In order to measure the total neutron cross-section, two Li-glass scintillator detectors with different characteristics were prepared. One was of the ${ }^{6} \mathrm{Li}$ enriched type ( $>90 \%$, GS20) and the other was of the ${ }^{7} \mathrm{Li}$ enriched type (>99.99\%, GS30). Since the neutron detection by the Li-glass scintillator is effected by the ${ }^{6} \mathrm{Li}(\mathrm{n}, \alpha) t$ reaction, the enrichment of the ${ }^{7} \mathrm{Li}$ isotopes hinders the neutron detection efficiency. The low neutron detection efficiency of the GS30 scintillator allows its usage for estimating the amount of $\gamma$-ray background. Two different volumes of Li-glass, $1 \mathrm{~mm} \times 50 \mathrm{~mm} \times 50 \mathrm{~mm}$ ( $1 \mathrm{~mm}$ thickness $)$ and $10 \mathrm{~mm} \times 100 \mathrm{~mm} \times 100 \mathrm{~mm}(10 \mathrm{~mm}$ thickness $)$, were prepared for each of the scintillators. Compared to the $1 \mathrm{~mm} \mathrm{Li}$-glass, the $10 \mathrm{~mm}$ one has higher efficiency for both high energy neutrons and background $\gamma$-rays. In order 


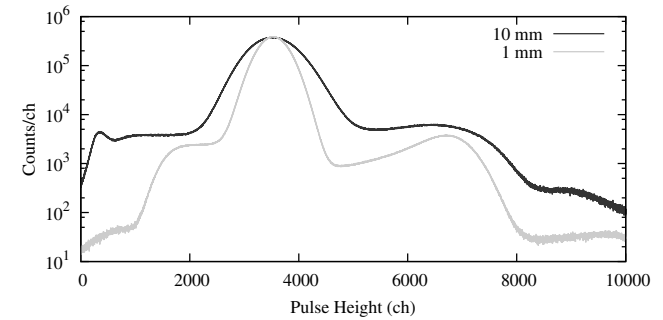

Figure 4. Pulse height spectrum of a GS20-type neutron detectors. The grey solid line represents the $1 \mathrm{~mm}$ thick scintillator, and the red solid line the $10 \mathrm{~mm}$ thick one.

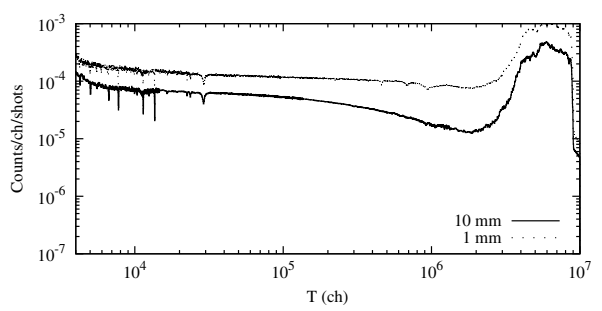

Figure 5. The time-of-flight spectra for different thicknesses of GS20 scintillators. The dashed line shows the time-of-flight spectrum of the $1 \mathrm{~mm}$ thick scintillator, compared with the $10 \mathrm{~mm}$ thick one (solid line).

to reduce $\gamma$-ray background along the beam duct pipe, $5 \mathrm{~cm}$ or $10 \mathrm{~cm}$ lead bricks were located at the upstream side of samples.

Figure 4 shows the pulse height spectrum registered by the GS20 scintillator. The $1 \mathrm{~mm}$ scintillator has better energy resolution than the $10 \mathrm{~mm}$ one because of its higher light-collection efficiency. Figure 5 shows the time-offlight spectrum of a blank target run using a GS20 with both $1 \mathrm{~mm}$ and $10 \mathrm{~mm}$ thickness. The count rate value is normalized by the J-PARC proton beam power. For the $10 \mathrm{~mm}$ scintillator, the counting rate was limited by a narrow collimator in order to reduce the detection dead time.

For neutron detection using the Li-glass detector, the main background is due to $\gamma$-rays. In order to remove the effect of $\gamma$-rays, two different methods were investigated. One method was to fit the neutron detection peak and background spectrum by appropriate likelihood functions for each time-of-flight bin. The neutron detection counts can be deduced from the area of the peak fitting function. Another method is to use the GS30 scintillator. As mentioned above, the GS30 scintillator has less sensitivity to neutrons but almost the same efficiency for the $\gamma$-rays compared to the GS20 scintillator. From the measurement in the same experimental setting for both GS20 and GS30, the $\gamma$-ray background is estimated from the GS30 spectrum.

Figure 6 shows an example of the GS30 $\gamma$-ray background estimation using neutron black resonance filters in the ANNRI facility. Neutron events in the GS20 detector disappear in the filtered location. The bottom points of black resonances represent $\gamma$-ray background. The spectrum obtained by the GS30 scintillator reproduces the $\gamma$-ray background shape clearly.

The fitting method is also checked by the same condition. Figure 7 shows the difference between the GS20

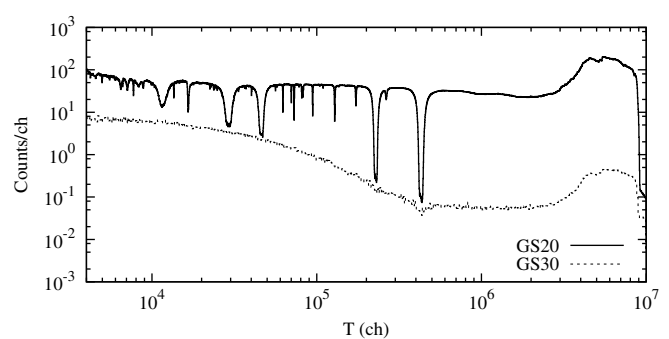

Figure 6. The $\gamma$-ray background estimation using the GS30 scintillator. The solid line shows the time-of-flight spectrum by the GS20 scintillator and the dashed line means the GS30 scintillator. The black filter resonance positions can be reproduced by the GS30 spectrum.

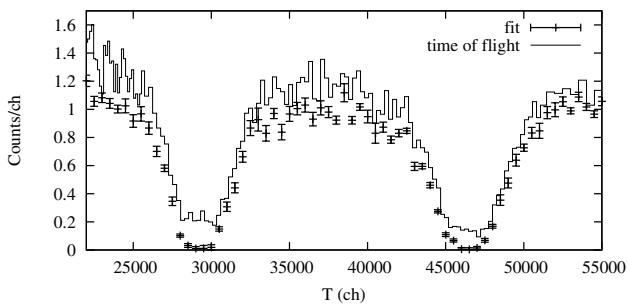

Figure 7. The fitted area counts of the neutron detection events. The solid line corresponds to the event-count time-of-flight distribution. Points with error bars represent the peak area counts with fitting errors.

time-of-flight spectrum and the peak area counts of the fitted function. The peak area counts go to zero at the location of the black resonance.

\subsection{Count rate performance}

In order to estimate the count rate efficiency of the DAQ system, the constant dead-time analysis method was applied. In the constant dead-time analysis, it is assumed that there is a dead-time only in the region within a fixed time distance from the dead-time source.

Let $p_{i}$ be the probability of the $i$ th channel in the timeof-flight spectrum being treated as a dead-time source, and $N$ be the fixed time distance. The probability $p_{o}$ for the detector being dead in the 0th channel is written as

$$
1-p_{0}=\prod_{i=1}^{N}\left(1-p_{i}\right)
$$

The CAEN v1724 ADC works with fixed dead-time for each signal even if the signal is not treated as a valid event (defined as "not invalid"). In case that the signal is treated as invalid (defined as "double pulses were detected, but could not distinguish pulse height information each other"), the time-stamp information is recorded anyway. In order to estimate the count rate efficiency by the constant dead-time analysis method, we can use this time-stamp information as a starting point of the constant dead-time. In this case, the probabilities of the dead-time appearance are proportional to the real signal rates.

On the other hand, the CAEN v1720 ADC limits the starting points of the dead-time only for the triggered 

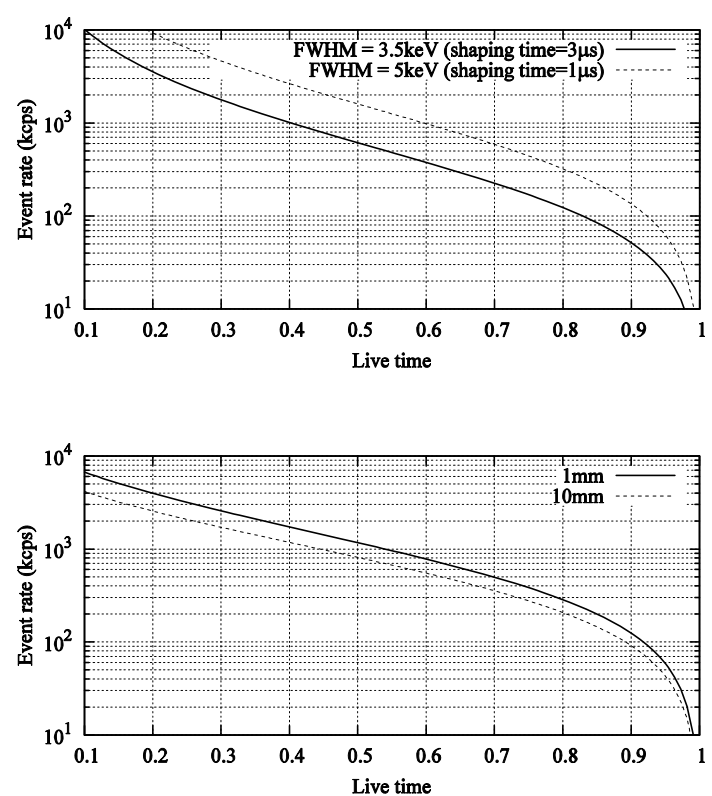

Figure 8. The counting efficiency of the DAQ system. The upper figure shows the acceptable event rate of the Ge detectors with different shaping time. The solid line represents the $3 \mu$ s shaping time setting. The dashed line shows the $1 \mu \mathrm{s}$ case. The lower figure shows the difference of the counting rate capability of the $1 \mathrm{~mm}$ thick (solid line) and the $10 \mathrm{~mm}$ thick (dashed line) Li-glass scintillators.

situation. In such case, the probabilities $p_{i}$ must be given proportional to the number of triggers.

The pile-up rejection gate, which is applied by offline software analysis, always rejects events by probabilities proportional to the real signal rates. Taking these different behaviours into account carefully, the counting efficiency of the DAQ system was determined.

Figure 8 shows the counting efficiency. The upper figure depicts the Ge detector with different shaping time settings. The lower figure shows the difference between $1 \mathrm{~mm}$ thick and $10 \mathrm{~mm}$ thick Li-glass. Typically, the system works with $50 \mathrm{kcps}$ for the Ge system and $100 \mathrm{kcps}$ for the Li-glass system.

\section{Summary}

A new DAQ system for the J-PARC/MLF/ANNRI facility was developed. The system was designed based on the client-and-server model for the flexibility of an increase in the number of ADCs. The CAEN v1724 100-MHz 14-bit ADC and v1720 250-MHz 12-bit ADC were used for acquiring data from the $\mathrm{Ge}$ detectors and Li-glass detectors, respectively. The applicability of the time-offlight method was checked for both Ge and Li-glass detectors. The ADC non-linearity, energy resolution, and coincidence analysis performances were checked for the Ge detector system. Two methodologies, peak fitting, and a GS30 scintillator, were tested for $\gamma$-ray background removal for the Li-glass detector. The counting rate performance was about $50 \mathrm{kcps}$ for the Ge system and $100 \mathrm{kcps}$ for the Li-glass system with $90 \%$ live-time.

The authors would like to thank the accelerator and technical staff at J-PARC for operating the accelerator and the neutron production target and for their other experimental support. The present study includes the result of "Research and Development for accuracy improvement of neutron nuclear data on minor actinides" entrusted to the Japan Atomic Energy Agency by the Ministry of Education, Culture, Sports, Science and Technology of Japan (MEXT).

\section{References}

[1] M. Harada, S. Meigo, M. Ito, E. Dantsuji, K. Takagiwa, H. Takada, F. Maekawa, M. Futakawa, M. Nakamura, Y. Miyake, Y. Ikeda, Nucl. Instrm. Meth. A 600, 87-90 (2009)

[2] MLF Annual Report 2014 (2015)

[3] M. Igashira, Y. Kiyanagi and M. Oshima Nucl. Instrm. Meth. A 600, 332-334 (2009)

[4] Nucl. Sci. Technol. 49, 708 (2012)

[5] http://www.caen.it/

[6] K. Shibata, O. Iwamoto, T. Nakagawa, N. Iwamoto, A. Ichihara, S. Kunieda, S. Chiba, K. Furutaka, N. Otuka, T. Ohsawa, T. Murata, H. Matsunobu, A. Zukeran, S. Kamada, and J. Katakura, J. Nucl. Sci. Technol. 48(1), 1-30 (2011) 